\title{
Repos et suspens artificiels. Otium et écriture dans « la nouvelle d'une ivresse de haschich » de Walter Benjamin
}

Repose and Artificial Suspense. Otium and Writing in "the Novella of a Hashich Intoxication" of Walter Benjamin

\section{Robert Krause}

\section{OpenEdition}

\section{Journals}

Édition électronique

URL : https://journals.openedition.org/recherchestravaux/821

DOI : 10.4000/recherchestravaux.821

ISSN : 1969-6434

\section{Éditeur}

UGA Éditions/Université Grenoble Alpes

\section{Édition imprimée}

Date de publication : 1 juin 2016

Pagination : 131-140

ISBN : 978-2-84310-325-4

ISSN : 0151-1874

\section{Référence électronique}

Robert Krause, «Repos et suspens artificiels. Otium et écriture dans « la nouvelle d'une ivresse de haschich » de Walter Benjamin », Recherches \& Travaux [En ligne], 88 | 2016, mis en ligne le 01 janvier 2017, consulté le 29 octobre 2021. URL : http://journals.openedition.org/recherchestravaux/821 ; DOI : https://doi.org/10.4000/recherchestravaux.821 


\section{Repos et suspens artificiels. Otium et écriture dans «la nouvelle d'une ivresse de haschich» de Walter Benjamin}

Myslowitz-Braunschweig-Marseille, publié en I930 dans une revue allemande, est «la nouvelle d'une ivresse de haschich». Pour des raisons évidentes, il y a un rapport entre le thème de la drogue et l'écriture dans ce récit littéraire de Walter Benjamin, qui s'inscrit dans le contexte biographique de l'auteur. Autour de l'année 1927, Benjamin commence à expérimenter lui-même différentes drogues et à prendre des notes qu'il considère comme très importantes ${ }^{1}$. L'occasion lui est donnée par une invitation d'Ernst Joël et de Fritz Fränkel, deux médecins et amis de jeunesse, qui lui proposent d'expérimenter le haschich, l'opium et la mescaline. Le philosophe Ernst Bloch participe également à plusieurs séances. Pour la production littéraire de Benjamin, une prise de haschich qui a lieu le 29 septembre 1928 à Marseille est tout particulièrement décisive. Le compte rendu méticuleux qu'il en retire livre la matière de son récit Myslowitz - Braunschweig - Marseille ainsi que celle d'un article intitulé "Haschich à Marseille», publié dans la Frankfurter Zeitung du 4 décembre 1932 et traduit en français pour Les Cahiers $d u$ Sud (n ${ }^{\circ}$ I68) en janvier $1935^{2}$. Ces trois textes ne sont donc pas seulement liés par un rapport thématique, mais aussi par

I. Voir sa lettre à G. Scholem du 30 janvier 1928, dans W. Benjamin, Gesammelte Briefe, vol. III, éd. Ch. Gödde, Francfort-sur-le-Main, Suhrkamp, I997, p. 324. Ces notes se trouvent dans W. Benjamin, Gesammelte Schriften, vol. VI, éd. R. Tiedemann et R. Schweppenhäuser, Francfort-sur-le-Main, Suhrkamp, 1985, p. 558-618. (Désormais GS.)

2. W. Benjamin, «Haschich à Marseille», dans Euvres, vol. II, trad. de l'allemand M. de Gandillac, R. Rochlitz et P. Rusch, Paris, Gallimard, 200o, p. 48-58. Voir M. Schneider, "Drogenprotokolle», dans B. Lindner (dir.), Benjamin-Handbuch, Leben - Werk - Wirkung, Stuttgart-Weimar, Metzler, 2006, p. 676-678, p. 676. 
un rapport intertextuel et génétique. Le sujet et l'expérience qu'ils ont en commun sont l'ivresse du haschich, représentée comme une situation de repos et de suspens, une situation d'otium. Si l'on prend l'ivresse au sérieux ${ }^{3}$, il est alors évident que le haschich peut engendrer un état propice à l'expérience de l'oisiveté4. Pour mieux montrer et étudier cette représentation de l'ivresse et de sa fonction narrative chez Benjamin, nous nous livrerons à une analyse de son récit Myslowitz - Braunschweig - Marseille sur trois planss.

Le premier relève de la construction narrative de la nouvelle et étudie plus particulièrement le récit cadre du début de l'histoire. On s'intéressera ensuite au lieu dans lequel se déroule le récit enchâssé, à la représentation de la ville de Marseille. Sur un troisième plan, il faut prendre en compte l'expérience du haschich elle-même, ainsi que la manière dont elle est décrite dans la nouvelle.

\section{L'esprit de l'otium comme source de la narration}

La nouvelle Myslowitz - Braunschweig - Marseille comprend deux parties narratives, un récit cadre et un récit enchâssé. Le premier est très bref et commence par un refus d'endosser la responsabilité auctoriale du texte : le narrateur extradiégétique déclare que ce n'est pas lui qui a inventé l'histoire qui va suivre, mais un peintre inconnu.

Cette histoire n'est pas de moi. Le peintre Eduard Scherlinger, que je vis pour la première et la dernière fois le soir où il la raconta, était-il ou non un grand conteur, je ne m’appesantirai pas là-dessus, car en cette époque de plagiats, se trouvent toujours quelques auditeurs qui veulent vous attribuer une histoire, justement quand on explique qu'on ne fait que la rapporter fidèlement. Mais je l'ai entendue dans un des rares lieux classiques que Berlin recèle pour les conteurs ou les auditeurs potentiels, un soir, chez Lutter \& Wegener $[s i c]^{6}$.

3. Pour une analyse de la théâtralité des états d'ivresse chez Benjamin, voir B. Marschall, Die Droge und ihr Double. Zur Theatralität anderer Bewußtseinszustände, Cologne-Weimar-Vienne, Böhlau, 2000, p. 179-268. Pour les rapports avec le concept d'ivresse chez Nietzsche, Bataille et Adorno, voir C. Bäuerl, Zwischen Rausch und Kritik I. Auf den Spuren von Nietzsche, Bataille, Adorno und Benjamin, Bielefeld, Aisthesis, 2003.

4. Voir L. Fuest, Poetik des Nicht(s)tuns. Verweigerungsstrategien in der Literatur seit I80o, Munich-Paderborn, Fink, 2008, p. I09-II8.

5. La version originale allemande de ce texte se trouve dans W. Benjamin, Gesammelte Schriften, vol. IV/2, éd. R. Tillmann, Francfort-sur-le-Main, Suhrkamp, 1982, p. 729-737. Jusqu'à maintenant il n'existe qu'une traduction française de cette nouvelle, qui figure dans l'ouvrage Rastelli raconte et autres récits, trad. Ph. Jaccottet, Paris, Seuil, 1987, p. 39-53, sous le titre «Myslowice - Braunschweig - Marseille, une histoire de haschich». Toutes les citations en langue allemande figurant dans la présente contribution sont traduites par S. Goldblum, que je remercie aussi pour son aide et pour la relecture de cet article.

6. GS, IV/2, p. 729 . 
Un tel refus de la responsabilité auctoriale n'a en soi rien d'étonnant; on le retrouve par exemple chez Hoffmann et plus généralement dans la littérature romantique, que Benjamin, qui a écrit en I918-I9I9 sa thèse de doctorat sur Le concept de critique de l'art dans le Romantisme allemand, connaît bien. Hoffmann, Heine et les autres auteurs berlinois du Romantisme tardif se réunissaient souvent chez Lutter \& Wegner, un café doublé d'une cave à champagne situé à proximité de la place du Marché des Gendarmes, le Gendarmenmarkt. La mention de cet endroit gastronomique célèbre contribue à créer un «effet de réel $^{7}$ » qui donne au lecteur l'impression que le récit décrit un monde réellement existant. De plus, la mention de ce café souligne l'importance d'un cadre social qui implique une première situation de repos et d'oisiveté : on est assis à une table, au sein d'un petit groupe de personnes. On a raconté et parlé ensemble, maintenant les conversations se dispersent ${ }^{8}$. Dans cette ambiance détendue, le philosophe Ernst Bloch prend la parole. Son intervention est spectaculaire :

C'est alors $[. .$.$] que mon ami, le philosophe Ernst Bloch, lança cette phrase : il$ n'y a personne dit-il, qui une fois dans sa vie n'ait manqué d'un cheveu de devenir millionnaire. [...] Plus nous nous penchions sur cette affirmation et plus nous prenions plaisir à en débattre, pour finalement l'un après l'autre devenir pensifs, car chacun pensait à la fois où il avait été, dans sa vie, le plus proche des millions?.

Le fait de mentionner le nom d'Ernst Bloch, un des plus proches amis de Benjamin dans les années 1920, relève aussi de l'effet de réel. Si on la prend comme une information contextuelle, cette mention vient créer, du moins de manière embryonnaire, un pacte autobiographique ${ }^{\mathrm{I}}$. De plus, cette référence n'est pas dénuée d'ironie; en effet Bloch était alors en train de développer sa pensée critique, inspirée du marxisme. Dans ce contexte, le fait qu'il se préoccupe des différentes manières d'accomplir ou non sa vocation de millionnaire constitue au moins un contre-emploi. En outre, le penseur de l'utopie en est dans la nouvelle réduit à défendre des rêves de petit-bourgeois qui cadrent mal avec la hauteur de vue de sa philosophie ${ }^{\mathrm{II}}$.

Le narrateur extradiégétique a beaucoup de points communs avec l'auteur Benjamin. Ce que le personnage de Bloch dit dans la nouvelle donne lieu à une réflexion de la part de ceux qui l'écoutent. Pour prouver la justesse de sa thèse, ils commencent à raconter des histoires personnelles. Eu égard à cette

7. Voir R. Barthes, «L'effet de réel», Communications, n II, I968, p. 84-89.

8. Voir GS, IV/2, p. 729.

9. Ibid.

Io. Voir P. Lejeune, Le Pacte autobiographique, Paris, Seuil, 1975.

II. Pour une analyse contextuelle du "jeu de masques et de variations" entre Benjamin et Bloch, voir A. Allerkamp, «Du "philosopher surréaliste”. Myslowice - Braunschweig Marseille", Cahiers de L'Herne, n IO4, Benjamin, 2013, p. 217. 
ambiance détendue et chaleureuse, on peut constater que c'est l'otium, qui, dans ce contexte, fait naitre la narration.

Sans raison apparente, le narrateur choisit l'histoire d'Eduard Scherlinger. Autant que possible, il essaie de reproduire fidèlement le récit, tel qu'il l'a reçu du peintre lui-même ${ }^{\mathrm{r} 2}$. De cette manière, Scherlinger est introduit comme narrateur intradiégétique. Ce changement est par ailleurs marqué par l'emploi des pronoms personnels, qui marque nettement le passage d'une instance narrative à une autre.

\section{Marseille, destination de voyage, lieu de la flânerie}

C'est le peintre Scherlinger qui est le personnage principal et le narrateur - intradiégétique - du récit enchâssé. Au début il raconte qu'il a hérité une petite fortune qu'il a utilisée pour partir dès que possible en voyage en France. Marseille est la destination finale de son voyage et doit en constituer le point culminant :

Quand, après la mort de mon père, $[\ldots]$ je me retrouvai à la tête d'une fortune appréciable, je précipitai mon départ pour la France. Surtout, j'étais heureux de découvrir, avant même la fin de la vingtaine, la ville de Marseille, la patrie de Monticelli, à qui je devais tout mon art; sans parler de toutes les autres choses que Marseille représentait pour moi à cette époque ${ }^{13}$.

De multiples raisons expliquent le choix de Scherlinger. En tant que peintre, il éprouve une grande curiosité pour Marseille, la ville natale et le domicile d'Adolphe Monticelli (I824-I886), peintre français d'origine italienne. Mais il fait aussi allusion à un intérêt plus secret, voire intime pour Marseille, dont on peut supposer, au vu du reste de la nouvelle, qu'il a un lien avec le haschich. En effet, la vieille ville portuaire, qui unit l'Europe et l'Afrique, l'Occident et l'Orient, terre d'origine du haschich, semble être un lieu prédestiné pour s'échapper du quotidien et de ses devoirs, et pour expérimenter «les paradis artificiels", d'autant que cette ville était aussi un des plus grands comptoirs au monde pour le commerce du chanvre, qui servait à la fabrication des cordages $^{14}$. En outre, Scherlinger se prépare à partir pour longtemps. Il met en ordre ses affaires en confiant son héritage à une banque. Pour le cas où une

I2. Voir GS, IV/2, p. 729.

I3. Ibid.

I4. Une représentation de Marseille comportant des traits similaires se trouve chez J. Roth, «Die weißen Städte», dans Werke, vol. II : Das journalistische Werk I924-I928, éd. K. Westermann, Cologne, Kiepenheuer \& Witsch, I990, p. 45I-502, 50I-502. 
bonne occasion se présenterait, le partenaire junior de la banque lui promet de l'avertir tout de suite ${ }^{\text {Is. }}$. Pour dire les choses autrement, Scherlinger part pour le loisir (otium), et laisse à un autre le soin de s'occuper de ses affaires (negotium).

Dans ce cadre, la banque demande un mot de passe à Scherlinger pour se protéger contre un éventuel abus. Du point de vue narratif, la mention de l'abus fonctionne comme une prolepse. Elle constitue un renvoi discret à la suite de l'histoire dans laquelle l'ivresse du haschich est indiquée comme un abus de médicaments. Choisir un mot de passe pose des problèmes inattendus à Scherlinger, la quête d'un nom qui soit en adéquation avec la situation étant comparée aux jeux de la mascarade et de la fausse identitér ${ }^{16}$. On retrouve ce motif de l'identité changeante dans la suite de l'histoire, à Marseille. Ni la physionomie de la ville portuaire ni l'identité culturelle du peintre ne sont évidentes. À partir de son arrivée à Marseille, on remarque des incohérences :

Vers midi, un jour pesant de juillet, j'arrivai à la gare Saint-Louis de Marseille, après une halte de quatre semaines à Paris. Des amis m'avaient indiqué l'hôtel Regina, non loin du port; je me laissai à peine le temps d'y prendre une chambre, de vérifier que la lampe de chevet et les robinets étaient en état de marche et je me mis en chemin $^{17}$.

D'une part, Scherlinger évoque sa pause d'un mois à Paris et la chaleur estivale qui règne à Marseille. D'autre part, il a hâte de partir tout de suite de l'hôtel et de se lancer à la découverte de la ville. Conformément à la règle qu'il s'est fixée pour ses voyages, Scherlinger veut explorer la banlieue avant de se rendre dans

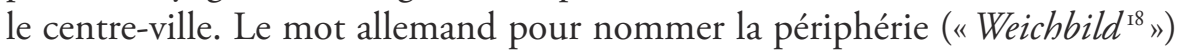
et la description des contrastes très marqués entre le paysage provençal et la végétation de la côte maritime font référence à la physionomie polyvalente de Marseille. La longue rue de Lyon est présentée comme la porte d'entrée qui ouvre sur un mélange détonnant. Les noms des quartiers, des rues et des boutiques sont comparés à l'explosion de mines représentée par l'énumération suivante qui évoque explicitement l'explosion linguistique à laquelle Marseille confronte le visiteur :

La longue rue de Lyon est le couloir de mines que Marseille a creusé dans le paysage pour le faire exploser à Saint-Lazare, Saint-Antoine, Arenc et Septèmes et pour le recouvrir des éclats d'obus des langues de tous les peuples et de toutes les entreprises : Alimentation Moderne, Rue de Jamaïque, Comptoir de la Limite,

I5. Voir GS, IV/2, p. 729-730.

16. Voir ibid., p. 730.

17. Ibid.

I8. Ibid. 
Savon Abat-Jour, Minoterie de la Campagne, Bar du Gaz, Bar Facultatif. Et par là-dessus, la poussière composée de sel marin, de calcaire et de paillettes de mica' ${ }^{19}$.

La variété culturelle et commerciale de la ville portuaire lui confère son caractère polyphonique et polyglotte. Cette polyphonie se retrouve aussi dans le récit littéraire de Benjamin, où la curiosité et l'admiration pour l'ambiance méditerranéenne sont omniprésentes. Marseille est le lieu vibrant de la flânerie du protagoniste-narrateur. Autour du vieux port, l'atmosphère change une fois de plus. Maintenant Scherlinger ressent une ambiance euphorique et fraternelle :

Si j'avais jusqu'alors cheminé seul dans les faubourgs les plus populeux, à partir de là je me sentis impérieusement intégré dans le défilé des marins d'humeur festive, des dockers rentrant chez eux, des ménagères en promenade qui, ponctué d'enfants, se mouvait le long des cafés et des bazars, pour se perdre peu à peu dans les rues adjacentes et atteindre seulement en la personne de quelques mariniers ou flâneurs, comme moi, la grande artère principale, la rue des commerces, des bourses et des étrangers, la Cannebière $[s i c]^{20}$.

C’est le hasard qui dirige les pas du flâneur, ou le mouvement que lui imposent les forces de la rue. La rue la plus célèbre et la plus emblématique de Marseille, la Canebière, inspire les rêveries du protagoniste-narrateur. Bientôt se présente une nouvelle situation de repos. Elle coïncide avec le moment où Scherlinger, tout à ses réflexions, arrive à l'endroit prototypique du loisir : le passage ${ }^{21}$. Là, dans le Passage de Lorette, il remarque la présence d'hommes et de femmes ensommeillés. Le monde entier lui semble être réduit à un seul dimanche après-midi, le jour férié par excellence ${ }^{22}$. À cet instant le protagoniste est pris de tristesse et commence une métaréflexion sur l'écriture et la narration. Ce n'est pas un hasard si Benjamin introduit l'épisode de la prise de haschich par l'évocation de la Canebière; en effet le nom de cette rue renvoie au cannabis, au chanvre dont on faisait le commerce à Marseille ${ }^{23}$. Cette analogie entre la rue et la drogue est renforcée par l'orthographe fautive de Benjamin, qui écrit "Cannebière» avec deux «n", comme cannabis.

Cela ajouterait, dit Scherlinger en souriant, un beau vernis romantique, si je décrivais maintenant comment je me suis procuré le haschich dans un quelconque bar portuaire mal famé de la ville, par un Arabe, qui aurait pu être chauffeur sur un

19. Ibid., p. 73I.

20. Ibid.

2I. Voir R. M. Schaper, Der gläserne Himmel. Die Passagen des I9. Jahrhunderts als Sujet der Literatur, Francfort-sur-le-Main, Athenäum, 1988.

22. Voir GS, IV2, p. 732.

23. Voir A. Bles, Dictionnaire historique des rues de Marseille, Marseille, Jeanne Laffitte, 1989, p. 98. Je remercie S. Goldblum pour cette trouvaille qui est tout à fait en accord avec l'intérêt de Benjamin pour les étymologies et pour les noms propres. 
cargo ou portefaix. Mais je n'ai nul besoin de ce vernis, car je ressemblais peutêtre plus à ces Arabes qu'aux étrangers que leur chemin conduit dans ce genre de bars. Dans la mesure du moins où j'emportais mon haschich avec moi dans mes voyages ${ }^{24}$.

Dans cette fantaisie, le narrateur intradiégétique fait explicitement référence aux topoï romantiques. De façon ludique, il met son histoire et sa propre identité culturelle en question. Scherlinger commence à inventer une suite d'histoire possible, mais il se débarrasse tout de suite de cette fantaisie. Ce faisant, il opère un jeu complexe avec les codes de l'exotisme. En effet, si la possibilité de faire naître un sentiment d'étrangeté chez le lecteur par l'évocation de lieux interlopes et de la rencontre de personnages venus d'ailleurs est envisagée, cette dernière est aussitôt révoquée au profit d'un autre moteur. En effet, c'est le narrateur Scherlinger lui-même qui se met en scène comme figure exotique en insistant sur ses points communs avec les marins arabes. Cette comparaison crée le lien entre les voyages et le haschich, ce qui nous ramène par un chemin de traverse à un topos de l'exotisme et à la thématique centrale de la nouvelle.

\section{Le flâneur enivré}

Scherlinger, le protagoniste-narrateur, est familier du haschich. D'une part, il a des connaissances théoriques et déclare connaître tous les témoignages littéraires de l'ivresse, de Baudelaire (Les Paradis artificiels) à Hermann Hesse (Le Loup des steppes) ${ }^{25}$. D'autre part, Scherlinger a l'habitude d'avoir son haschich avec lui quand il part en voyage.

Ce soir-là à Marseille, dans sa chambre d'hôtel, Scherlinger consomme $\mathrm{du}$ haschich. La raison qu'il en donne est remarquable : pour lui, prendre de la drogue participe de la volonté de pénétrer encore plus profondément dans l'atmosphère magique de la ville ${ }^{26}$. Si l'on envisage l'usage de substances psychoactives, il s'agit d'une situation de suspens : Scherlinger est allongé sur son lit, il fume du haschich et lit un texte dont le titre n'est pas précisé. Il est sûr que personne ne va le déranger dans ses rêveries, mais croyant cela, il fait erreur. L'effet de la drogue se fait attendre ${ }^{27}$. En revanche, Scherlinger entend

24. GS, IV/2, p. 732.

25. Ibid., p. 732. À ce sujet, voir A. Kupfer, Göttliche Gifte. Kleine Kulturgeschichte des Rausches seit dem Garten Eden, Stuttgart-Weimar, Metzler, 1996; du même, Die künstlichen Paradiese. Rausch und Realität seit der Romantik. Ein Handbuch, Stuttgart-Weimar, Metzler, 1996.

26. Voir GS, IV/2, p. 732.

27. Voir ibid. 
la sonnette. Quelqu'un arrive avec une dépêche de sa banque, qui lui propose une affaire commerciale digne d'intérêt. À ce moment-là, Scherlinger se rend compte de l'heure qu'il est. Cette indication nous donne à voir que sa situation d'otium lui a fait oublier l'heure. Il doit maintenant réagir, mais il est inquiet et mécontent. La dépêche est la figuration concrète de l'incursion du negotium, qui dérange la situation de $\operatorname{loisir}^{28}$.

Pour aller au bureau de poste et envoyer un télégramme à sa banque, Scherlinger entreprend une promenade nocturne. Hanté par la peur d'oublier le mot de passe, il presse le pas $^{29}$. À diverses erreurs de jugement, il remarque que l'effet de la drogue n'est pas aussi faible qu'il l'avait initialement cru. Scherlinger veut régler ses affaires le plus vite possible, mais sous l'emprise du haschich, tandis qu'il marche dans les rues, il oublie le but de sa sortie. Deux types de loisir, la flânerie et l'ivresse du haschich, se réunissent dans la suite de l'histoire. Le lecteur y trouve une véritable phénoménologie de l'ivresse : elle commence avec des éclats de rire exaltés ou heureux. Ensuite, la personne enivrée éprouve un état de bien-être assorti à une perception spatiotemporelle spécifique :

Voici que se font valoir les exigences temporelles et spatiales propres au consommateur de haschich. Elles sont, c'est bien connu, absolument royales. Versailles, pour qui a pris du haschich, n'est pas trop grand, ni l'éternité trop longue ${ }^{3 \circ}$.

La dimension métaphorique mérite notre attention. La classification des exigences ("royales») conduit à l'image du château de Versailles et de l'éternité. La royauté se présente comme une forme de dépassement de la bourgeoisie artiste à laquelle appartient Scherlinger vers la noblesse qui se dégage de son personnage. Ici, il se retrouve dans un état d'exception qui fait du bourgeois héritier un roi et transforme sa finitude en éternité, toutes choses qui sont caractéristiques de l'oisiveté :

Et sur la toile de fond des dimensions gigantesques du vécu intérieur, de la durée absolue et de l'incommensurable espace, un humour merveilleux s'attarde d'autant plus volontiers avec ce sourire béat sur le caractère infiniment contestable de tout étant ${ }^{31}$.

Il y a un lien permanent entre l'ivresse et la flânerie. Le protagoniste marche, il se déplace avec facilité, il se sent à l'aise et décidé à la fois. La dimension

28. Voir $G S, I V / 2$, p. 733.

29. Voir ibid.

30. Ibid., p. 734.

3I. Ibid. 
esthétique, l'impression de la beauté de toute chose est nettement marquée par le texte, qui ne craint pas l'hyperbole, elle-même teintée d'ironie ${ }^{32}$.

Mais notre flâneur enivré oublie son but. Assis à la table d'un café, au calme, il ressent l'effet singulier du haschich. Là, Scherlinger regarde les gens qui l'entourent et tout à coup, il commence à en étudier la physionomie ${ }^{33}$. Dans cette situation de repos, il voit une fantasmagorie d'une petite ville de l'Est, Myslowitz en Pologne. Le climax de l'histoire est atteint quand Scherlinger accède à une fulgurante connaissance de lui-même. Il éprouve une illusion d'omniscience dans une situation de suspens :

Mon regard se posa sur les plis que formait mon pantalon de plage blanc, je les reconnus, les plis du burnous; mon regard se posa sur ma main, je la reconnus, brune, éthiopienne, et alors que mes lèvres hermétiquement fermées restaient collées l'une à l'autre, refusant tant la boisson que les paroles, un sourire monta de l'intérieur jusqu'à elles, un sourire altier, africain, sardanapalien, le sourire de l'homme qui est sur le point de percer à jour le cours du monde et le destin et pour lequel ni les choses ni les noms n'ont plus aucun secret. Je me voyais assis là, brun et silencieux. Braunschweiger ${ }^{34}$.

La pointe de ce passage repose sur un jeu de mots difficile à rendre en français, qui vient rompre abruptement l'effet d'exotisme construit par Benjamin dans les lignes qui précèdent. Le personnage est qualifié de "brun" (braun) et de "silencieux» (schweigend), ces deux éléments étant repris dans le composé Braunschweiger, qui évoque immédiatement un habitant de la ville de Brunswick, et plus, un prince éthiopien.

L'otium dans l'ivresse du haschich se manifeste comme une condition de possibilité de la constitution du sujet tout à la fois énonciateur d'une narration et capable d'un retour réflexif sur lui-même. Dans la nouvelle de Benjamin, c'est une situation de loisir qui donne lieu à des réflexions portant sur l'harmonie du rapport que le sujet entretient avec le monde. Cette harmonie correspond à la fin de l'histoire. Le protagoniste s'est endormi, il a oublié d'aller au bureau de poste. Quand il se réveille, Scherlinger entend les informations sur l'opération financière qu'il a manquée. Mais déçu ou agacé, il est heureux ${ }^{35}$. Scherlinger a vécu une situation de loisir exceptionnelle qui vaut plus que la fortune qu'il aurait pu obtenir. Et son histoire nous montre bien que les paradis artificiels, les récits et protocoles sur les drogues de Benjamin ainsi que les écrits d'autres auteurs traitant de ce sujet, méritent l'attention des chercheurs. En effet, la drogue, par la reconfiguration de la perception qu'elle permet, offre une vision

\footnotetext{
32. Voir ibid.

33. Voir ibid., p. 735.

34. Ibid., p. 737.

35. Voir ibid.
} 
du monde, de la temporalité et du sujet toute particulière. C'est en cela que ce sujet peut être le vecteur de développements sur d'autres sujets, comme ici l'oisiveté, la flânerie et l'exotisme.

Cet effet de la drogue devient encore plus évident si l'on prend en compte les autres textes que Benjamin a consacrés au haschich, à savoir son article «Haschich à Marseille» et ses notes sur les expériences collectives qui témoignent de sa curiosité quasi scientifique pour les drogues ${ }^{36}$. De plus, ces protocoles mériteraient encore un éclairage de la part de la philologie benjaminienne ${ }^{37}$.

36. Dans cet interêt quasi scientifique, les textes de Benjamin sur le haschich rencontrent ceux de Baudelaire (Le Poème du hachisch, Du vin et du hachisch). À ce sujet, voir E. S. Burt, "Baudelaire and Intoxicants", dans R. Lloyd (dir.), The Cambridge Companion to Baudelaire, Cambridge, Cambridge University Press, 2006, p. II7-I29; Th. Klinkert, "Wissenschaft als Quelle poetischer Inspiration. Baudelaires Poetik des Rausches", dans H. Hufnagel et O. Krämer (dir.), Das Wissen der Poesie. Lyrik, Versepik und die Wissenschaften im I9. Jahrhundert, Berlin-Boston, De Gruyter, 20I5, p. I07-I2I.

37. Voir M. Schneider, «Drogenprotokolle», art. cité, p. 677; B. Marschall, Die Droge und ihr Double, ouvr. cité, p. I79. 